\title{
Differential expression of CD44 variants among meningioma subtypes
}

\author{
S O Suzuki, T Iwaki, T Kitamoto, M Mizoguchi, M Fukui, J Tateishi
}

\begin{abstract}
Aims/background-CD44 is a widely distributed cell surface molecule which has numerous isoforms generated by alternative splicing. The diverse functions related to the CD44 variants (CD44v) have been reported in various physiological and pathological conditions. The pattern of expression of CD44v among meningioma subtypes was investigated to ascertain whether CD44 variants play a role in a variety of biological processes, such as epithelial differentiation and extracranial metastasis.
\end{abstract}

Methods-Twenty three meningiomas were studied immunohistochemically using novel antibodies directed against CD44 isoforms. Six of the 23 samples were analysed by reverse transcription polymerase chain reaction (RT-PCR), followed by Southern blotting with CD44v specific probes.

Results-In meningothelial, fibrous and anaplastic meningiomas, a standard form of CD44 was detected by RT-PCR and was homogeneously expressed in tumour cells when studied immunohistochemically. CD44v was not detected in these subtypes. In secretory meningiomas, however, CD44v isoforms were strongly expressed in the cell clusters that produce secretory granules and also accumulated in the granules. The population of tumour cells immunopositive for CD44v was similar to that which stained with antibodies directed against carcinoembryonic antigen, epithelial membrane antigen and ezrin. On RT-PCR with Southern blotting, only the secretory type showed high level expression of CD44v.

Conclusions-CD44v in meningiomas is expressed in relation to tumour cell differentiation towards the epithelial type. (F Clin Pathol: Mol Pathol 1996;49:M140-M146)

Keywords: CD44, meningioma, cell differentiation.

Correspondence to: Dr S O Suzuki Department of

Neuropathology,

Neurological Institute,

Faculty of Medicine, Kyushu University,

Fukuoka, 812-82 Japan.

e-mail:

yan@np.med.kyushu-u.ac.jp

Accepted for publication 26 March 1996
CD44 is a widely distributed cell adhesion molecule which has numerous isoforms. CD44 variants $(\mathrm{CD} 44 \mathrm{v})$ are generated by insertion of alternatively spliced exons into a single site of standard CD44 (CD44s) mRNA, as well as by post-translational modification. ${ }^{\prime}$ CD44 has been reported to play a role in a variety of biological phenomena, such as haemopoiesis, ${ }^{2}$ inflammation, ${ }^{3}$ development, ${ }^{4}$ immune reactions, ${ }^{5}{ }^{6}$ and metastasis of tumour cells. ${ }^{7-9}$ Some CD44 isoforms are known to be expressed in cells capable of differentiating into epithelial cells, such as cells in the basal layer of the $\operatorname{skin}^{10}$ and those in squamous metaplasia of lung tissue. ${ }^{11}$

Meningioma is one of the most commonly encountered intracranial neoplasms. The tumour is thought to be of arachnoid cell origin and is classified into a variety of histological subtypes, ${ }^{12}$ each of which shows characteristic morphological and biological features. Other authors have reported a weak CD44 immunoreactivity ${ }^{13}$ and expression of a few variant forms on northern blotting ${ }^{14}$ in meningiomas. However, these studies did not describe either the histological subtype of the material examined or the detailed splicing pattern of the CD44 molecules; also, the function of CD44 in meningiomas was not discussed. To investigate whether CD44 isoforms play a role in the biology of meningiomas - for example, epithelial differentiation and metastatic ability, as documented in other types of tumour, we therefore studied the pattern of expression of CD44s and CD $44 \mathrm{v}$ in various types of meningiomas, including two anaplastic samples manifesting extracranial metastases, by means of immunohistochemistry and reverse transcription polymerase chain reaction (RT-PCR) followed by Southern blotting.

\section{Methods}

Brain tumours were obtained either at surgery or at necropsy, fixed in $10 \%$ neutral buffered formalin overnight and then embedded in paraffin wax. Twenty three meningiomas were studied, comprising five secretory meningiomas, seven meningothelial meningiomas, three fibrous meningiomas, six transitional meningiomas, and the original intracranial lesions and extracranial metastatic deposits of two anaplastic meningiomas manifesting an extracranial metastasis. Five samples of carcinomas of extracranial origin were taken from metastatic brain lesions (three lung adenocarcinomas, one colon adenocarcinoma and one renal cell carcinoma). Two adenoid cystic carcinomas which had invaded the skull were also sampled. These carcinoma samples, which frequently expressed CD44v, were mainly used to 
Table 1 Primer sequences

\begin{tabular}{ll}
\hline Primer & Sequence \\
\hline Cp-1 & GACACATATTGCTTCAATGCTTCAGC \\
Cp-2 & GATGCCAAGATGATCAGCCATTCTGGAAT \\
KN-1 & TCTTCAAATACCATCTCAGCA \\
KN-2 & GGTGCTGGAGATAAAATCTTC \\
SS-1 & GAAAAACTGAGGTGTCTGTC \\
KN-3 & ACACAAAACAGAACCAGGAC \\
KN-4 & TGGGGTCTCTTCTTCCTCAT \\
SS-2 & GTCATCCTTGTGGTTGTCT \\
KN-5 & GCTACCCAGAAGGAACAGT \\
KN-6 & CCTTCTTCCTGCTTGATGAC \\
SS-3 & AACTGTCCTCTGGGCTTGGT \\
KN-7 & ACAGGTTTGGTGGAAGATTTG \\
KN-8 & TGTCAGAGTAGAAGTTGTTGG \\
SS-4 & GTAGAAGTTGTTGGATGGTC \\
KN-9 & GGAAGAAGAGACCCAAATCAT \\
KN-10 & CTGATAAGGAACGATTGACAT \\
SS-5 & GTGTGGGTAATGAGAGGTAT \\
\hline
\end{tabular}

characterise the novel antibodies and probes used in the present study. Sections were stained routinely with haematoxylin and eosin. Some of the secretory meningiomas were also stained with periodic acid Schiff to confirm the diagnosis. Of the tissue samples examined immunohistochemically, 13 (one secretory, three meningothelial and two anaplastic meningiomas, five metastatic carcinomas of extracranial origin, and two adenoid cystic carcinomas) were immediately frozen for RNA extraction. Total RNA was extracted using the acid guanidium thiocyanate-phenol-chloroform method. ${ }^{15}$

\section{POLYMERASE CHAIN REACTION}

The primer oligonucleotide sequences used are summarised in table 1 and fig 1 and were designed according to the reported CD44 cDNA sequence. ${ }^{16}$ RT-PCR was performed as described previously ${ }^{17}$ using the CP-1/CP-2 primer pair. To clone the five domains which undergo alternative splicing, the PCR products were re-amplified with the following nested primer pairs: $\mathrm{KN}-1 / \mathrm{KN}-2, \mathrm{KN}-3 / \mathrm{KN}-4, \mathrm{KN}-5 /$ $\mathrm{KN}-6, \mathrm{KN}-7 / \mathrm{KN}-8$, or $\mathrm{KN}-9 / \mathrm{KN}-10$ for domains $1,2,3,4$, and 5 , respectively. The CD44s and nested PCR products representing each of the five domains were cloned into a pCR vector (TA cloning, Invitrogen, USA). Each clone was sequenced using the dideoxy chain termination method (data not shown).

DOMAIN SPECIFIC SOUTHERN HYBRIDISATION Using the cloned domain specific DNA fragments as cDNA probes, the patterns of expression of CD44v in the RT-PCR products described earlier were investigated by Southern blotting. Each domain specific cDNA was labelled with ${ }^{32} \mathrm{P}$ using internal primers (SS-1, SS-2, SS-3, SS-4, and SS-5 for domains 1, 2, 3, 4 , and 5 , respectively; table 1 ).

CDNA LIBRARY SCREENING

A human colon carcinoma cDNA library in the $\lambda Z A P$ II vector (Stratagene, USA) was screened using the plaque hybridisation method with a CD44v cDNA probe synthesised by PCR. The clone, $\mathrm{pBS} \lambda 19 / 2$, contained the alternatively spliced exons v3-v10 (exon domains 1-5) in addition to the standard region.

ANTIBODIES

We produced polyclonal antibodies directed against the CD44 molecule with an insertion in the variant region, v3-v10. The clone $\mathrm{pBS} \lambda 9 / 2$ was transformed into Escherichia coli using the prokaryotic vector $\mathrm{pFd}$-APP770 and the fusion protein $\mathrm{Fd}-\lambda 9 / 2$ was purified as described elsewhere. ${ }^{18} \mathrm{~A}$ rabbit was then immunised with $\mathrm{Fd}-\lambda 9 / 2$. The antibody was affinity purified with the fusion protein $\mathrm{Fd}-\lambda 9 / 2$, blotted onto the Millipore membranes (Nihon Millipore Ltd, Japan), ${ }^{19}$ and was then designated C228AP. The identity of the Fd- $\lambda 9 / 2$ fusion protein and the specificity of the C228AP antiserum were confirmed by western blotting. ${ }^{20}$ The fusion proteins Fd-St and Fd-Var, containing the standard region only and the variant region (v3-v10) only, respectively, were also produced. The antiserum directed against $\mathrm{Fd}-\lambda 9 / 2$ was affinity purified with $\mathrm{Fd}-\mathrm{St}$ or Fd-Var, and then each was absorbed with the reversed fusion protein. The resulting antisera were designated SAP and VAP, respectively. The former was intended to be reactive only with the standard region, and the latter to be reactive only with the variant region. The specificity of SAP and VAP was confirmed by western blotting and immunohistochemistry.

A monoclonal antibody directed against the standard region of human CD44 was purchased from Biodesign, USA. Monoclonal antibodies directed against CD44v, var v6 and var v10, which recognise the CD44v epitopes encoded by exons v6 and v10, respectively, were purchased from Bender MedSystems,

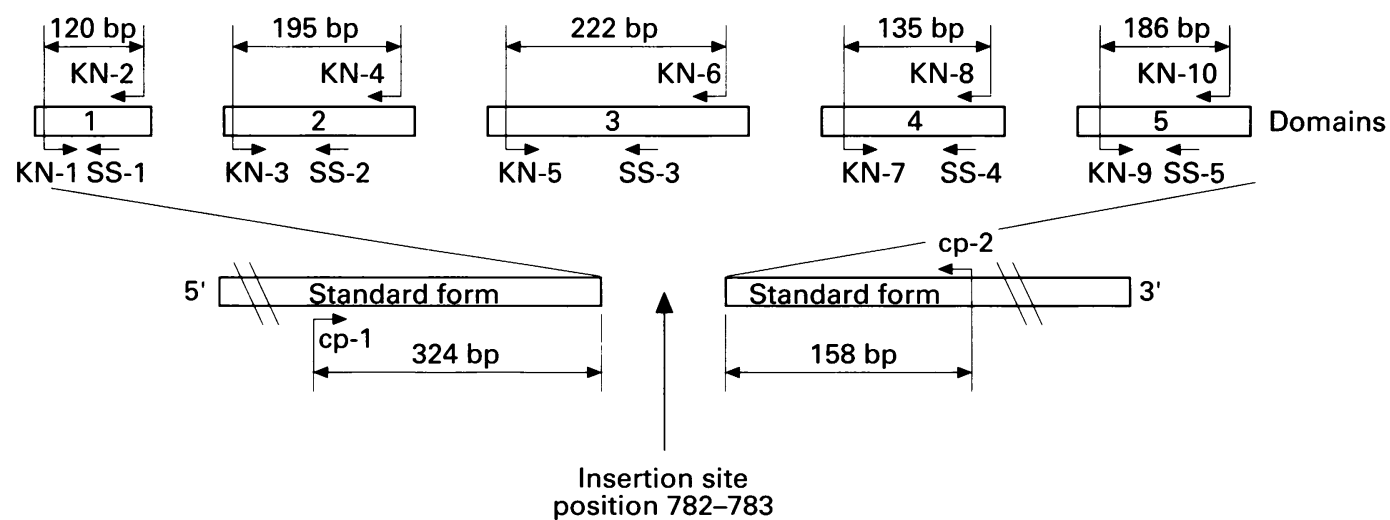

Figure 1 CD44 cDNA structure and the position of the primers. Each domain corresponds to the following exons: domain $1=v 3 ;$ domain $2=v 4$ and $v 5 ;$ domain $3=v 6$ and $v 7$; domain $4=v 8$ and $v 9$; and domain $5=v 10$. 


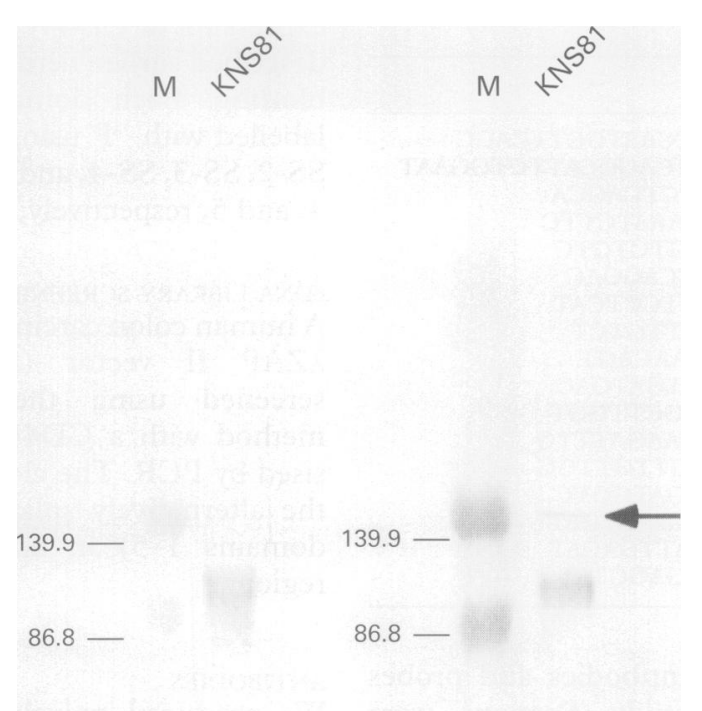

A

B

Figure 2 Characterisation of the C228AP polyclonal antiserum. KNS81 was lysed with a solution of $50 \mathrm{mM}$ Tris- $H C l, p H 6.8,0.5 \%$ Triton- $X, 2 \mathrm{mM}$ EDTA, and $2 \mathrm{mM}$ phenylmethylsulphonylfluoride (PMSF). The soluble fraction was tested with $(A)$ the monoclonal antibody directed against $C D 44 s$ and $(B) C 228 A P$. C228AP recognised bands identical with those labelled by the CD44s monoclonal antibody and also recognised an additional band with a higher molecular weight, which was thought to be of a CD44 splice variant (arrow). $M=$ molecular weight marker.

Austria. A monoclonal antibody directed against ezrin was obtained from Chemicon, USA. Monoclonal antibody directed against epithelial membrane antigen (EMA) was purchased from Dako (Glostrup, Denmark). Polyclonal antiserum directed against carcinoembryonic antigen (CEA) was purchased from IBL, Japan.

IMMUNOHISTOCHEMISTRY

The Streptavidin-biotin method was used as described previously. ${ }^{20}$ Paraffin wax sections
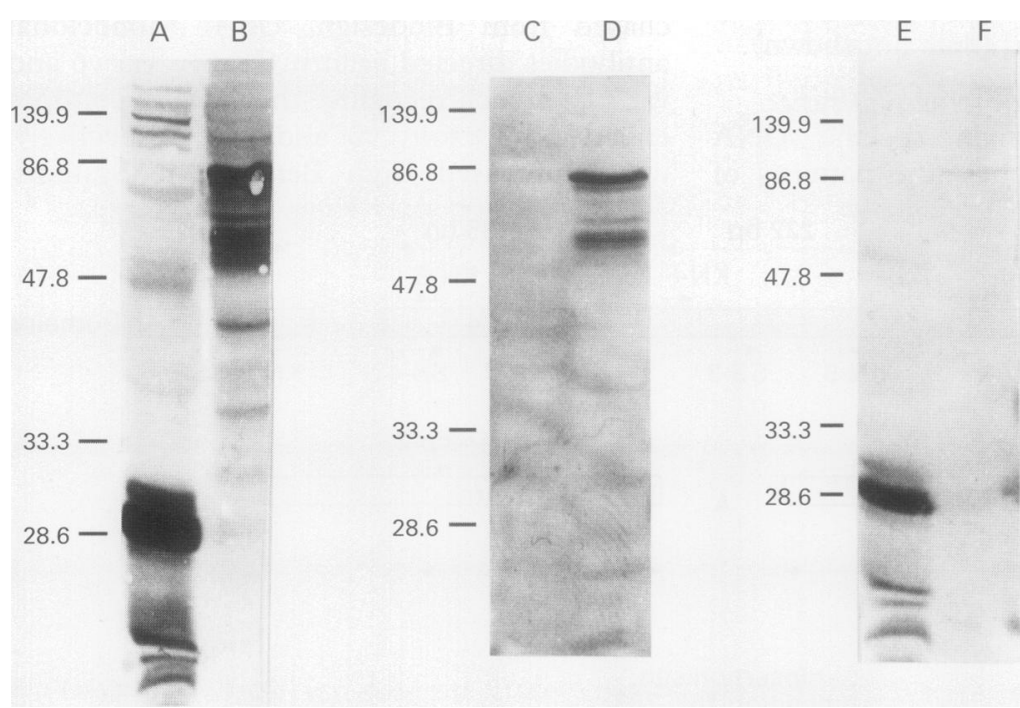

Figure 3 Specificity of C228AP, SAP and VAP. Immunoreactivity of C228AP ( $A$ and $B), V A P(C$ and $D)$ and $S A P(E$ and $F)$ against fusion proteins $F d-S t(A, C$ and $E$ ) and $F d$-Var $(B, D$ and $F)$. were pretreated in an autoclave to enhance antigen retrieval. ${ }^{20} 22$

\section{Results}

SPECIFICITY OF THE PROBES FOR SOUTHERN

BLOTTING

To confirm the specificity of the domain specific probes, the PCR products from cloned plasmids representing each domain were checked by hybridisation with the probes for every domain. There was no crosshybridisation among the domains (data not shown).

\section{WESTERN BLOTTING}

The $F d-\lambda 9 / 2$ fusion protein reacted with monoclonal antibodies directed against CD44s, CD44 var v6 and var v10. Fd-St and Fd-Var reacted with monoclonal antibodies directed against CD44s and var v10, respectively (data not shown). C228AP detected multiple bands with molecular weights of $80-120$ kilodaltons on western blotting of the soluble fraction of cultured human glioma cells KNS81, ${ }^{23}$ identical with those found with the commercial CD44 monoclonal antibody (fig 2). Furthermore, C228AP reacted with an additional higher molecular weight band of 165 kilodaltons, which was thought to be a splice variant of CD44 (fig 2). To confirm the epitope of C228AP, we compared the immunoreactivity of C228AP, SAP and VAP against the Fd-St and Fd-Var fusion proteins. C228AP detected both Fd-St and Fd-Var. SAP reacted strongly with Fd-St, but detected Fd-Var only faintly. By contrast, VAP reacted with Fd-Var, but not with Fd-St (fig 3). These findings indicated that C228AP was immunoreactive with both the standard and variant regions, and could be separated into two fractions, SAP and VAP. The former recognised the standard region and the latter the variant region without significant cross-reaction.

\section{IMMUNOSTAINING}

All five metastatic brain tumours showed positive staining with C228AP (fig 4A). Staining intensity varied from cell to cell. C228AP often showed preferential cell surface staining, which was similar to that on staining with VAP (fig 4B). By contrast, staining with SAP was rather homogeneous, and the intensity of the immunoreaction was almost the same across tumour cells (fig 4C). The staining pattern with SAP was similar to that with commercially supplied monoclonal antibody, which recognises CD44s only. Therefore, together with the findings of western blotting, we concluded that C228AP reacts with both the standard and variant regions, and that levels of expression of CD44v were highly heterogeneous across the metastatic carcinoma cells, which can be demonstrated by VAP immunostaining. Table 2 shows the expression of CD44v in each tumour type, as evaluated by VAP immunopositivity.

In meningothelial and fibrous meningiomas, tumour cells showed diffuse, weak staining or no staining at all with C228AP, as previously reported for CD44 expression in meningiomas. ${ }^{13}$ There was no significant staining in 
Table 2 CD44v expression as determined by VAP immunopositivity

\begin{tabular}{|c|c|c|c|}
\hline Tumour type & $\begin{array}{l}\text { Stongly } \\
\text { positive }\end{array}$ & $\begin{array}{l}\text { Weakly } \\
\text { positive }\end{array}$ & Negative \\
\hline \multicolumn{4}{|l|}{ Meningiomas $(n=23)$} \\
\hline secretory meningioma & 4 & 1 & 0 \\
\hline $\begin{array}{l}\text { meningothelial meningioma } \\
\text { meningioma }\end{array}$ & 0 & 0 & 7 \\
\hline fibrous meningioma & 0 & 0 & 3 \\
\hline transitional meningioma & 0 & 0 & 6 \\
\hline anaplastic meningioma & 0 & 0 & 2 \\
\hline \multicolumn{4}{|l|}{ Metastatic tumours $(n=7)$} \\
\hline lung adenocarcinoma & 3 & 0 & 0 \\
\hline colon adenocarcinoma & 1 & 0 & 0 \\
\hline renal cell carcinoma & 0 & 1 & 0 \\
\hline adenoid cystic carcinoma & 2 & 0 & 0 \\
\hline
\end{tabular}

characteristic structures of each meningioma type-that is, whorl formation in the meningothelial type or bundles of spindle shaped cells in the fibrous type. There was no difference in the staining intensity between the meningothelial and fibrous components of transitional meningiomas. In two anaplastic meningiomas, the original intracranial lesions and their extracranial metastatic deposits showed diffuse, weak or no staining with C228AP. There was no difference in either the staining pattern or signal intensity between common benign meningiomas and their malignant counterparts. All samples of secretory meningiomas showed strong C228AP immunopositivity in clusters of the cells producing PAS positive (fig 5A) secretory granules. These cell components, related to secretory granules, frequently showed strong membrane immunoreactivity, whereas other meningothelial tumour cells showed weak or no staining (figs 5B and $5 \mathrm{C}$ ). VAP stained these granule producing cells only (fig $5 \mathrm{D}$ ), whereas both the commercial monoclonal antibody, directed against the standard region, and SAP stained the tumour cell homogeneously, with no distinctive staining of the granule producing cells (figs $5 \mathrm{E}$ and $5 \mathrm{~F})$. These findings indicate that meningioma cells, regardless of their histological subtype, show diffuse expression of CD44s; the granule producing cells in secretory type express CD44v; and the other components of this type express CD44s only. The PAS positive secretory granules showed a variety of patterns of immunoreactivity with $\mathrm{C} 228 \mathrm{AP}$, ranging from negative to strongly positive. The CEA antiserum stained selectively those cells which were positive for VAP (fig 5G). EMA antibody also showed reactivity with those cells, but staining was strongest on the luminal surface of granule containing vesicles. Expression of ezrin, a recently reported CD44 associated undercoat constitutive protein, ${ }^{24}$ was diffuse and more intense than that of CD44 detected with C228AP in all meningioma subtypes. However, in three of five secretory meningiomas, the granule producing cells and the granules themselves were stained more intensely with the ezrin antibody than were the other cellular components (fig $5 \mathrm{H}$ ). ${ }^{13}$

RT-PCR AND SOUTHERN BLOTTING

CD44s, with a molecular size of 482 base pairs (bp) was detected in all the samples examined by RT-PCR (data not shown). On Southern
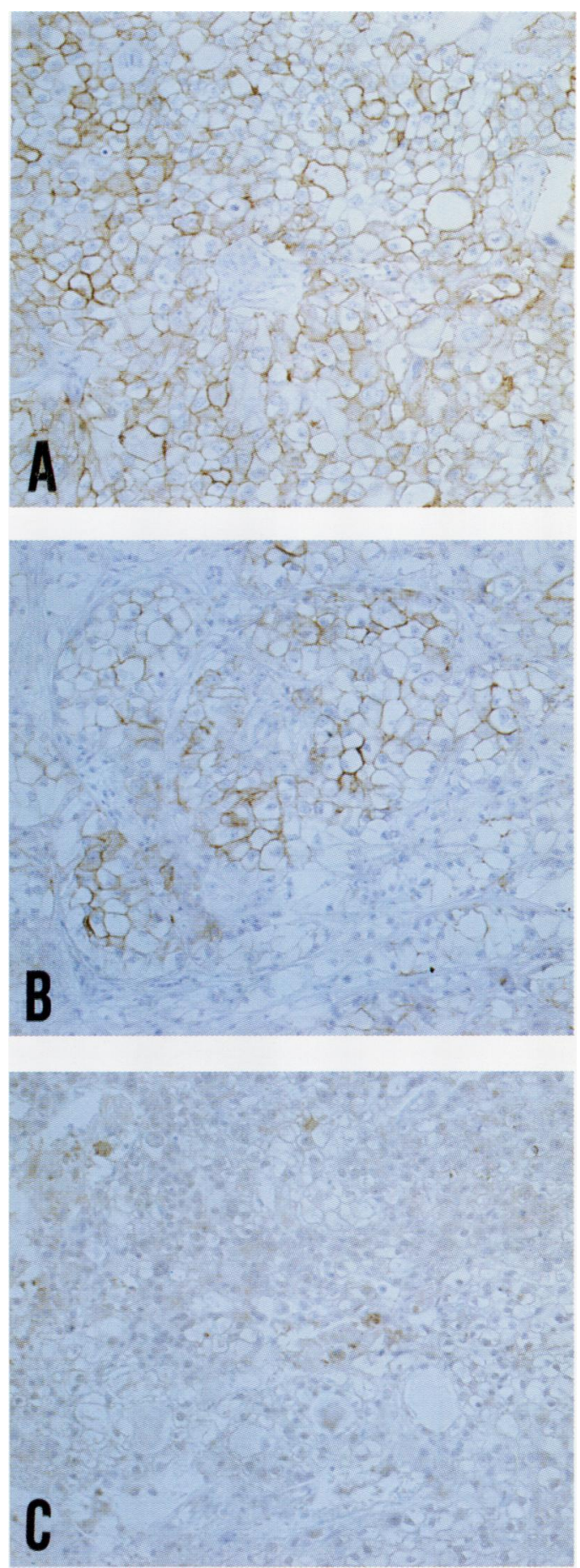

Figure 4 Immunohistochemical analysis of metastatic carcinomas. Immunostaining of metastatic brain lesion of adenocarcinoma for (A) C228AP (1:10, 50x), (B) VAP $(1: 5,50 x)$ and $(C) S A P(1: 2,50 x) . C 228 A P(A)$ and $V A P(B)$ stained the surfaces of the tumour cells intensely, while SAP (C) demonstrated weak homogeneous staining.

blots, all of the metastatic carcinoma samples showed expression of a variety of CD44v (table 3 ). Under the same hybridisation conditions all of meningothelial meningiomas expressed $\mathrm{CD} 44 \mathrm{v}$ on all domain specific hybridisation reactions although their molecular sizes were relatively smaller than those of metastatic carcinomas. A sample of secretory type meningioma showed the most excessive expression of large molecular size bands, including those over $1500 \mathrm{bp}$ in domain 2 (exons v4 and v5), 3 (exons v6 and v7), and 4 (exons v8 and v9) specific hybridisation reactions (table 3 ; fig 6 ). A primary lesion, lung metastasis and skin 

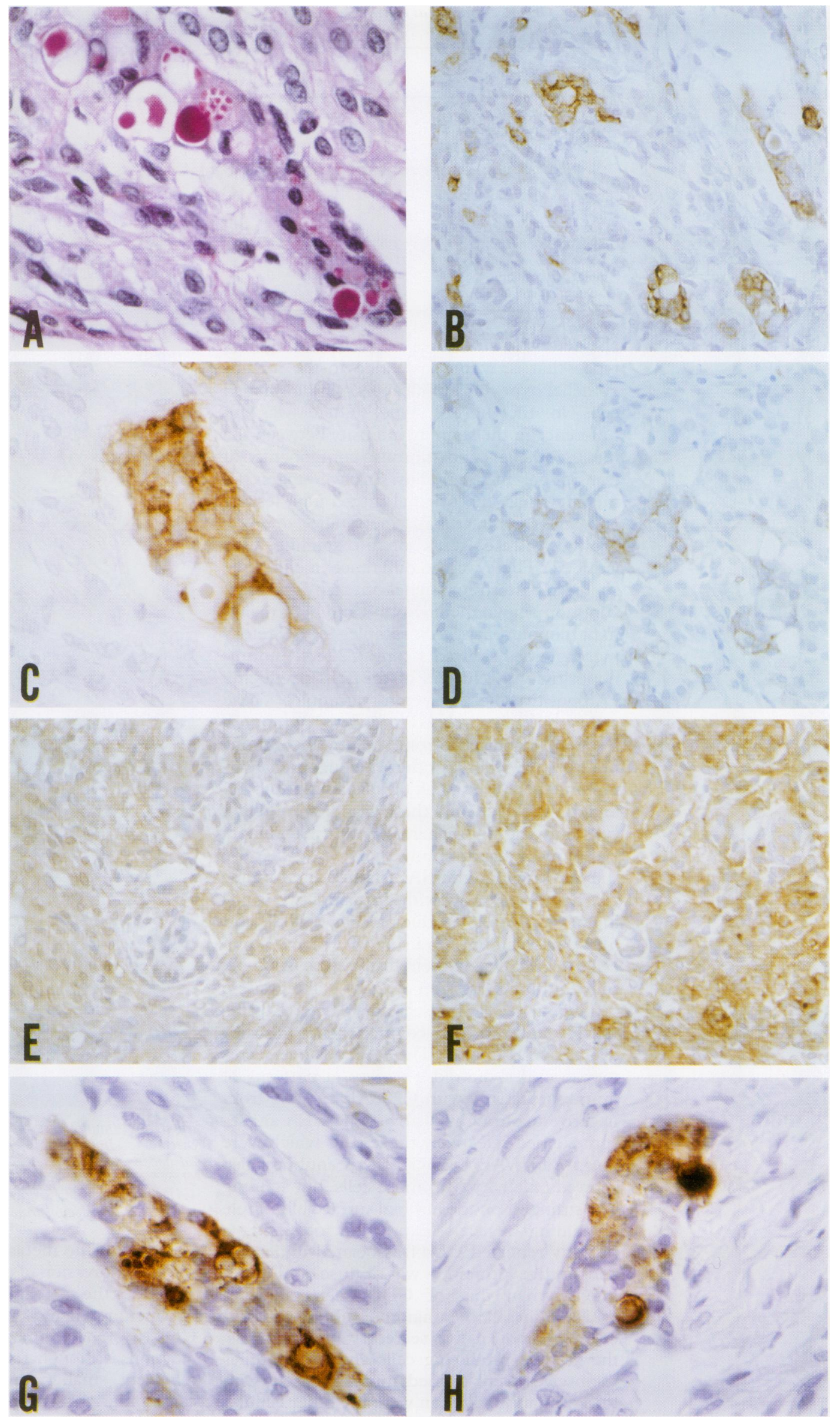

Figure 5 Immunohistochemical analysis of secretory meningiomas. (A) Section of secretory meningioma. PAS (200x). Immunostaining for $(B)$ and $(C) C 228 A P(1: 10,100 \times$ and $200 x$, respectively), (D) VAP $(1: 5,100 x),(E) S A P(1: 2$, $100 \times),(F)$ commercial monoclonal antibody directed against $C D 44 s,(G) C E A(1: 100,200 x)$, and $(H)$ ezrin $(1: 100$, 100X). The clusters of granule producing cells and the granules themselves were immunolabelled with C228AP, VAP and $C E A(B, C$ and $D)$. SAP $(E)$ and the commercial monoclonal antibody against $C D 44 s(F)$ showed homogeneous staining of tumour cells. The antibody directed against ezrin stained a more diffuse population of meningioma cells. However, $C D 44 v$ and CEA immunoreactive cells were stained more intensely than the other components $(H)$. 
Table 3 Expression patterns of CD44 splice variants $\geqslant 1500$ bp in size

\begin{tabular}{llllll}
\hline & \multicolumn{7}{l}{ Probe $(n)$} & & \\
\cline { 2 - 6 } Tumour type & Domain 1 & Domain 2 & Domain 3 & Domain 4 & Domain 5 \\
\hline Meningiomas $(\mathrm{n}=6)$ & & & & & \\
secretory meningioma & $0 / 1$ & $1 / 1$ & $1 / 1$ & $1 / 1$ & $0 / 1$ \\
meningothelial meningioma & $0 / 3$ & $0 / 3$ & $0 / 3$ & $0 / 3$ & $0 / 3$ \\
$\quad$ anaplastic meningioma & $1 / 2$ & $1 / 2$ & $0 / 2$ & $0 / 2$ & $0 / 2$ \\
Metastatic tumours (n=7) & & & & & \\
$\quad$ lung adenocarcinoma & $2 / 3$ & $2 / 3$ & $2 / 3$ & $2 / 3$ & $2 / 3$ \\
colon adenocarcinoma & $0 / 1$ & $1 / 1$ & $1 / 1$ & $1 / 1$ & $1 / 1$ \\
renal cell carcinoma & $0 / 1$ & $1 / 1$ & $1 / 1$ & $1 / 1$ & $1 / 1$ \\
adenoid cystic carcinoma & $2 / 2$ & $2 / 2$ & $2 / 2$ & $2 / 2$ & $2 / 2$ \\
\hline
\end{tabular}

$\mathrm{n}=$ Number of samples.

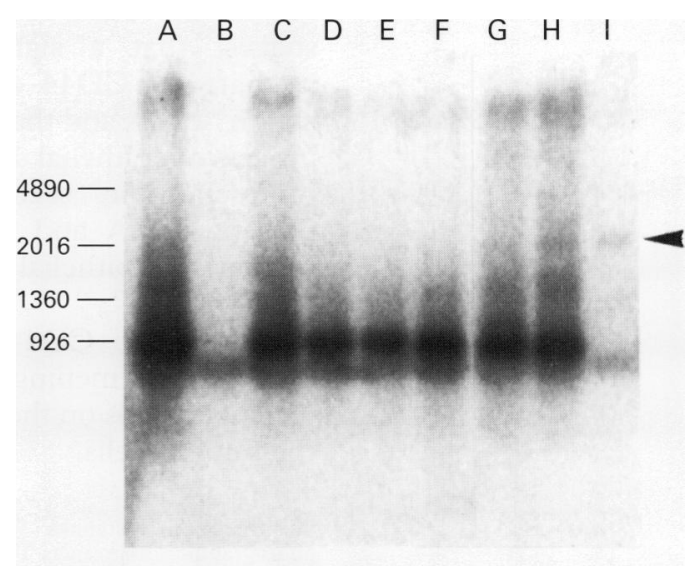

metastasis of anaplastic meningiomas produced large molecular size bands over $1500 \mathrm{bp}$ only on domain 1 (exon v3) and 2 (exons $v 4$ and v5) specific hybridisation (table 3 ; fig 6 ), while the other domain specific probes detected only those bands of relatively small molecular sizes as also seen in benign meningothelial meningiomas.

\section{Discussion}

In all meningioma subtypes we were able to detect the expression of CD44v as well as CD44s on RT-PCR, followed by Southern blotting. However, no significant difference in the level of expression was seen among the meningothelial, fibrous and anaplastic subtypes. To date, CD44v containing exon v6 has been discussed in relation to the metastatic potential of tumour cells. ${ }^{25}{ }^{26}$ However, on RT-PCR with Southern blotting, expression of the exon v6 containing domain in metastatic meningiomas closely resembled that of their benign counterparts. Immunostaining with VAP, which reacts only with CD44v, did not produce a response in anaplastic meningiomas. Therefore, in the present study, we were not able to elucidate the importance of expression of exon v6 for the metastatic potential of meningiomas. A recent study demonstrated that targeted disruption of the CD44 gene in a lymphosarcoma cell line had no effect on its high metastatic capacity, suggesting that not all lymphomas require CD44 for the metastatic process. ${ }^{27}$ It is possible that molecules other than CD44 play key roles in extracranial metastasis of anaplastic meningiomas. Further investigation of other candidate molecules as well as CD44 in a larger number of cases is warranted to elucidate the mechanisms underlying metastasis in meningiomas.

Significantly higher expression of CD44v was demonstrated both by immunohistochemistry and by RT-PCR with Southern blotting in secretory meningiomas than in samples of other subtypes. Secretory meningioma is a distinct form of meningioma that presents with numerous PAS positive hyaline inclusions under light microscopy and ordinarily manifests with a benign clinical course. ${ }^{28}$ The inclusion granules themselves and the cells producing the granules have been reported to be positive for some epithelial markers such as cytokeratin and EMA. ${ }^{29}{ }^{30}$ Electron microscopy studies showed that the granule containing cytoplasmic lumen is lined by well formed microvilli, which also constitute the secretory
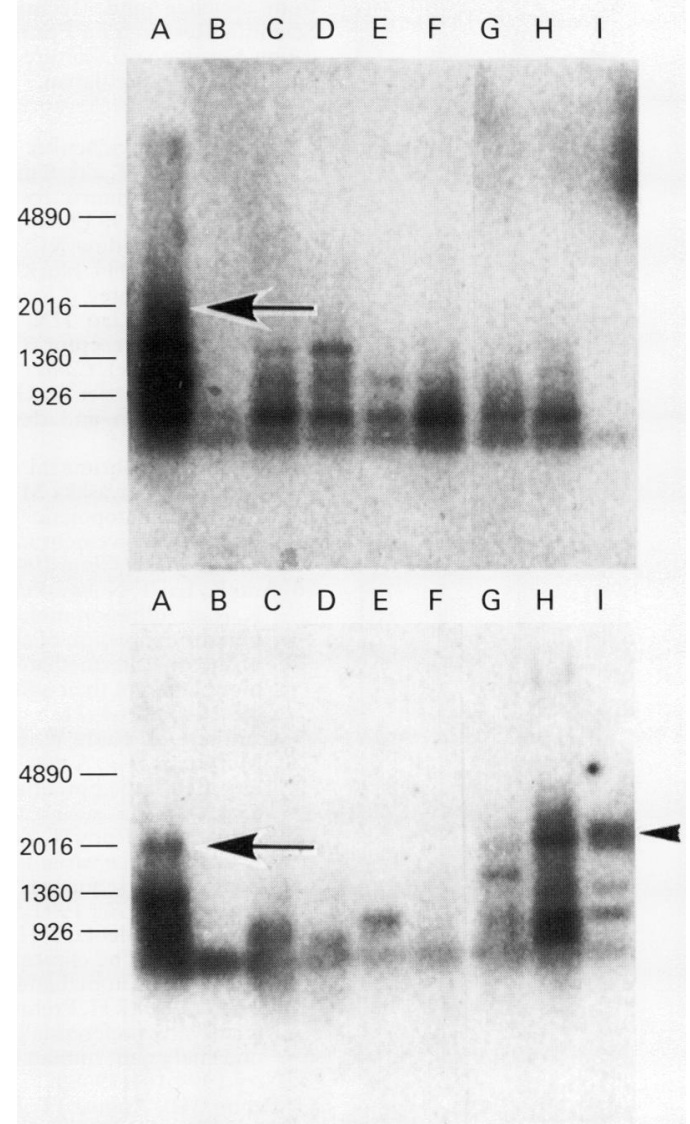

Figure 6 Domain 1 (upper), 3 (middle) and 2 (lower) specific hybridisation in meningiomas. (A) Surgical specimens of secretory meningioma; $(B)$ and $(C)$ surgical specimens of anaplastic meningioma; $(D-F)$ surgical specimens of meningothelial meningiomas; $(G-I)$ necropsy specimens from $B(G$, primary intracranial lesion; $H$, metastatic lung lesion; I, metastatic skin lesion). Excessive expression of $C D 44 v$ was observed in the secretory meningioma on all domain specific hybridisation reactions (arrows). CD44v expression was observed in the anaplastic meningiomas on hybridisation reactions only with domain 1 and 2 specific probes (arrowheads), but not with the other domain specific probes.

granules. ${ }^{28}{ }^{30}$ These findings indicate the occurrence of epithelial differentiation in the granule producing cells. Using immunohistochemistry, the expression of CD44v was shown to be restricted in the clusters of granule producing cells and their surrounding cells. Moreover, in three of five secretory meningiomas, the undercoat constitutive protein ezrin, which has been reported to play a role in microvillus formation ${ }^{31}$ and was recently found to interact with $\mathrm{CD} 44,{ }^{24}$ was also expressed intensely in granule producing cells. Prelim- 
inary studies of CD44 and ezrin expression suggest that a signal transduction system mediated by CD44-ezrin-actin filament interaction $^{24}$ exists and that this system is part of the process of epithelial differentiation and granule production in secretory meningiomas. At present, EMA and cytokeratin are the only markers of epithelial differentiation in meningioma cells. ${ }^{29} \quad 303233$ The present findings indicate that $\mathrm{CD} 44 \mathrm{v}$ is another molecule expressed in meningiomas, the expression of which depends on the differentiation activity of the tumour cells.

We thank K Hatanaka for technical and M Miura for photographical assistance. This study was supported by a grant from Science and Technology Agency, Grant-in-Aid for Scientific Research on Priority Area from the Ministry of Education Science and Culture, and a grant from the Ministry of Health and Welfare, Japan.

1 Jackson DG, Schenker T, Waibel R, Bell JI, Stahel RA. Expression of alternatively spliced forms of the CD44 Expression of alternatively spliced forms of the CD44 Int 7 Cancer 1994;8(Suppl): 110-15.

2 Miyake K, Medina KL, Hayashi S. Monoclonal antibodies to Pgp-1/CD44 block lymphopoiesis in long-term bone marrow cultures. $\mathcal{f}$ Exp Med 1990;171:477-88.

3 Haynes BF, Liao HX, Patton KL. The transmembrane hyaluronate receptor (CD44): multiple functions, multiple forms (review). Cancer Cells 1991;3:347-50.

4 Ruiz P, Schwarzler C, Günthert U. CD44 isoforms during differentiation and development. Bioassays 1995;17:1724 .

5 Toyama SN, Sorimachi H, Tobita Y, Kitamura F, Yagita $\mathrm{H}$, Suzuki K, Miyasaka M. A novel ligand for CD44 is serglycin, a hematopoietic cell lineage-specific proteoglycan cin, a hematopoietic cell lineage-specific proteoglycan. Possible involvement in lymphoid cell adh

6 Arai K, Iiai T, Nakayama M, Hasegawa K, Sato K, Ohtsuka $\mathrm{K}$, et al. Adhesion molecules on intermediate TCR cells. I Unique expression of adhesion molecules, CD44 + L-selectin, on intermediate TCR cells in the liver and the modulation of their adhesion by hyaluronic acid. Immunology 1995;84:64-71.

7 Günthert U, Rudy W, Reber S, Zoller M, Haussmann I, Matzku S, et al. A new variant of glycoprotein CD44 confers metastatic potential to rat carcinoma cells. Cell 1991; 65:13-24.

8 Hofmann M, Rudy W, Zoller M, Tolg C, Ponta H, Herrlich P. CD44 splice variants confer metastatic behavior in rats:
. P. CD44 splice variants confer metastatic behavior in rats: homologous sequences are expressed

9 Tarin D, Bolodeoku J, Hartfill SJ, Sugino T, Woodman AC, Yoshida $\mathrm{K}$. The clinical significance of malfunction of the CD44 locus in malignancy. $\mathcal{F}$ Neurooncol 1995;26:209-19.

10 Terpe HJ, Stark H, Prehm P, Günthert U. CD44 variant isoforms are preferentially expressed in basal epithelial of non-malignant human fetal and adult tissues. Histochemistry 1994;101:79-89.

11 Penno MB, August JT, Baylin SB, Mabry M, Linnoila RI, Lee VS, et al. Expression of CD44 in human lung tumors. Lee VS, et al. Expression of

12 Kleihues P, Burger PC, Scheithauer BW. Histological typing of tumors of the central nervous system. 2nd edn. Berlin: Springer Verlag, 1993.

13 Kuppner MC, Van ME, Gauthier T, Hamou MF, de Tribolet N. Differential expression of the CD44 molecule in human brain tumours. Int $\mathcal{F}$ Cancer 1992;50:572-7.
14 Harn HJ, Lee HS, Ho LI, Lee WH, Ding JH. Selective expression of CD44 messenger RNA splice variants in
four high grade human brain tumour cell lines. Biochem Mol Biol Int 1994;33:743-9.

15 Chomczynski P, Sacci N. Single-step method of RNA isolation by acid guanidium thiocyanate-phenol-chloroform extraction. Anal Biochem 1987;162:156-9.

16 Screaton GR, Bell MV, Jackson DG, Cornelis FB, Gerth U, Bell JI. Genomic structure of DNA encoding the lymphocyte homing receptor CD44 reveals at least 12 alternatively spliced exons. Proc Natl Acad Sci USA 1992; 89:12160-4.

17 Kitamoto T, Iizuka R, Tateishi J. An amber mutation of prion protein in Gerstmann-Sträussler syndrome with
mutant PrP plaque. Biochem Biophys Res Commun mutant PrP plaque.

18 Ohgami T, Kitamoto T, Weidmann A, Beyreuther K, Tateishi J. Alzheimer's amyloid precursor protein-positive degenerative neurites exist even within kuru plaques not specific to Alzheimer's disease. Am $f$ Pathol 1991; 139: $1245-50$

19 Iwaki T, Kume-Iwaki A, Liem R, Goldman J. $\alpha \mathrm{B}$-crystallin is expressed in non-leticular tissues and accumulates in Alexander's disease brain. Cell 1989;57:71-8.

20 Ohta M, Kitamoto T, Iwaki T, Ohgami T, Fukui M, Tateishi J. Immunohistochemical distribution of amyloid precursor protein during normal rat development. Dev Brain Res 1993;75:151-61.

21 Ohta M, Iwaki T, Kitamoto T, Takeshita I, Tateishi J, Fukui M. MIB1 staining index and scoring of histologic features in meningioma. Indicators for the prediction of biologic potential and postoperative management. Cancer 1994; 74:3176-89.

22 Shin RW, Iwaki T, Kitamoto T, Tateishi J. Hydrated autoclave pretreatment enhances Tau immunoreactivity in formalin-fixed normal and Alzheimer's desease brain tissues. Lab Invest 1991;64:693-702.

23 Takeshita I, Takaki T, Nakamura R, Maeyama R, Fukui M, Kitamura K. Established cell lines derived from human gliomas. Human Cell 1990;3:255-6.

24 Tsukita S, Oishi K, Sato N, Sagara J, Kawai A. ERM family members as molecular linkers between the cell surface glycoprotein CD44 and

25 Matsumura Y, Tarin D. Significance of CD44 gene products for cancer diagnosis and disease evaluation. Lancet 1992;340:1053-8

26 Wielenga VJ, Heider KH, Offerhaus GJ, Adolf GR, van den Berg FM, Ponta H, et al. Expression of CD44 variant proteins in human colorectal cancer is related to tumor progression. Cancer Res 1993;53:4754-6.

27 Driessens MHE, Stroeken PJM, Erena NFR, van der Valk $M A$, van Rijthoven EAM, Roos E. Targeted disruption of CD44 in MDAY-D2 lymphosarcoma cells has no effect on subcutaneous growth or metastatic capacity. $\mathcal{F}$ Cell Biol 1995;131:1849-55.

28 Alguacil GA, Pettigrew NM, Sima AA. Secretory meningioma. A distinct subtype of meningioma. Am f Surg Pathol 1986;10:102-11.

29 Ejeckam GC, Azadeh B, Hamad A. Secretory meningioma. Histopathology 1992;21:475-7.

30 Adachi J, Kazumoto K, Uki J, Takeda F, Kurozumi M, Nakazato Y. Secretory meningioma: a case report with immunohistochemical and ultrastructural study. Brain Tumor Pathology 1993;10:93-8.

31 Takeuchi K, Sato N, Kasahara H, Funayama N, Nagafuchi A, Yonemura S. Perturbation of cell adhesion and microvilli formation by antisense oligonucleotides to ERM family members. 7 Cell Biol 1994;125:1371-84.

32 Meis JM, Ordonez NG, Burner JM. Meningiomas: an immunohistochemical study of 50 cases. Arch Pathol Lab Med 1986;110:934-7.

33 Schnitt SJ, Vogel H. Meningiomas: diagnostic value of immunoperoxidase staining for epithelial membrane antigen. Am F Surg Pathol 1986;10:640-9. 